\title{
The RhoGAP crossveinless-c links trachealess and EGFR signaling to cell shape remodeling in Drosophila tracheal invagination
}

\author{
Véronique Brodu and Jordi Casanova ${ }^{1}$ \\ Institut de Biologia Molecular de Barcelona (CSIC) and Institut de Recerca Biomèdica, Parc Científic de Barcelona, \\ 08028 Barcelona, Spain
}

A major issue in morphogenesis is to understand how the activity of genes specifying cell fate affects cytoskeletal components that modify cell shape and induce cell movements. Here, we approach this question by investigating how a group of cells from an epithelial sheet initiate invagination to ultimately form the Drosophila tracheal tubes. We describe tracheal cell behavior at invagination and show that it is associated with, and requires, a distinct recruitment of Myosin II to the apical surface of cells at the invaginating edge. We show that this process is achieved by the activity of crossveinless-c, a gene coding for a RhoGAP and whose specific transcriptional activation in the tracheal cells is triggered by both the trachealess patterning gene and the EGF Receptor (EGFR) signaling pathway. Our results identify a developmental pathway linking cell fate genes and cell signaling pathways to intracellular modifications during tracheal cell invagination.

[Keywords: Morphogenesis; tracheal invagination; EGFR signaling; RhoGAP; acto-myosin; Drosophila]

Received December 12, 2005; revised version accepted April 24, 2006.

Organ formation requires coordinated changes in cell populations in terms of their proliferation, migration, differentiation, and shape. These synchronized changes are controlled by the genes specifying cell fate and by the ability of cells to respond to extracellular cues. This is achieved by means of signaling mechanisms that elicit cellular responses in terms of morphology and/or gene activation. Thus, the translation of signaling inputs and gene expression programs into definite cell changes is a crucial step in organ formation, a process finally shaped by changes in the cytoskeleton.

A model to approach this problem is the development of the Drosophila tracheal system, which provides a system to study the formation of organs consisting of complex tubular structures such as the lungs, kidney, and the vascular system. The tracheal system of Drosophila is a complex tubular network that conducts oxygen from the exterior to the internal tissues. It arises from the tracheal placodes, clusters of ectodermal cells that appear at each side of 10 embryonic segments, from the second thoracic segment to the eighth abdominal segment. Tracheal cells are specified by the activity of a set of genes whose expression in those cells is controlled by the genes that specify positional cues along the embryonic body axes

${ }^{1}$ Corresponding author.

E-MAIL jcrbmc@cid.csic.es; FAX 34-93-4034979.

Article is online at http://www.genesdev.org/cgi/doi/10.1101/gad.375706.
(Isaac and Andrew 1996; Wilk et al. 1996; Boube et al. 2000). Cells of each cluster invaginate and migrate in stereotyped and different directions to form each of the primary tracheal branches (for review, see Manning and Krasnow 1993). The general conclusion from many studies is that the direction of migration of the tracheal cells relies on a set of positional signals provided by nearby cells (Sutherland et al. 1996; Llimargas and Casanova 1997; Vincent et al. 1997; Wappner et al. 1997; Chihara and Hayashi 2000; Llimargas 2000). In addition, the establishment of interactions between tracheal cells and their substrates is a crucial step in tracheal cell migration, a process ultimately determined by molecules expressed at their surface (Franch-Marro and Casanova 2000; Boube et al. 2001).

Much is known about the genes required for the determination of the tracheal cells and for the initiation of the process of tracheal morphogenesis (Ghabrial et al. 2003). However, invagination and early migration are poorly characterized at the cellular level and not much is known about how the different genes and signaling pathways governing it eventually give rise to the cell changes that takes place at these stages. Understanding tracheal cell invagination is not only important because it accounts for the first step of tubulogenesis but also because invagination of epithelial tissues is a common process used to create multiple tissue layers.

In this work we investigate the cell shape changes that 
occur during early tracheal morphogenesis. We have found that tracheal invagination begins by apical constriction in a small group of cells that begin internalization followed by distinct rearrangements of the adjacent cells in the dorsal and ventral part of the placode. We have analyzed how this process is regulated by the activity of the trachealess (trh) transcription factor and EGF Receptor (EGFR) signaling. We also show that the spalt (sal) transcription factor down-regulates EGFR signaling in the dorsal side of the tracheal placode, and that this modulation of EGFR signaling is required for the organized invagination of the tracheal cells. We determine that tracheal invagination is associated with a distinct recruitment of Myosin II to the apical surface in the cells of the invaginating edge and that Myosin II is required for the proper invagination of tracheal cells. We have identified crossveinless-c $(c V-c)$, a gene coding for a RhoGTPase-Activating Protein (RhoGAP), as a key intermediate in this process. Altogether, our results unveil a developmental pathway, linking genes specifying cell fate and signaling pathways with cytoskeleton modifications that underlie early tracheal cell shape remodeling.

\section{Results \\ Local apical constriction initiates cell reorganization in tracheal invagination}

Tracheal cells are singled out as cell clusters in the ectodermal unicellular layer, one at each side of 10 central embryonic segments. We have focused our study on the central tracheal placodes because the first and last one have distinct features (Manning and Krasnow 1993). By stage 10 , tracheal cells form a flat epithelium with their neighboring ectodermal cells. Longitudinal optical sections (1 $\mu \mathrm{m}$ apart) show the apical cell membrane, visualized by $\mathrm{PKC}$, in a more exterior plane and the tracheal nuclei in a deeper one (Fig. 1A). A transverse optical section across the middle of the placode reveals its straight surface (Fig. 1B). By early stage 11, a group of around six cells reduces its apical cellular perimeter; this is the earliest indication of tracheal invagination since we can detect the constricted apical surface of those cells deeper inside (Fig. 1C). Local constriction is associated with cell shape changes, as those cells pinch at their apical surface while their basal surface and nuclei appear deeper than

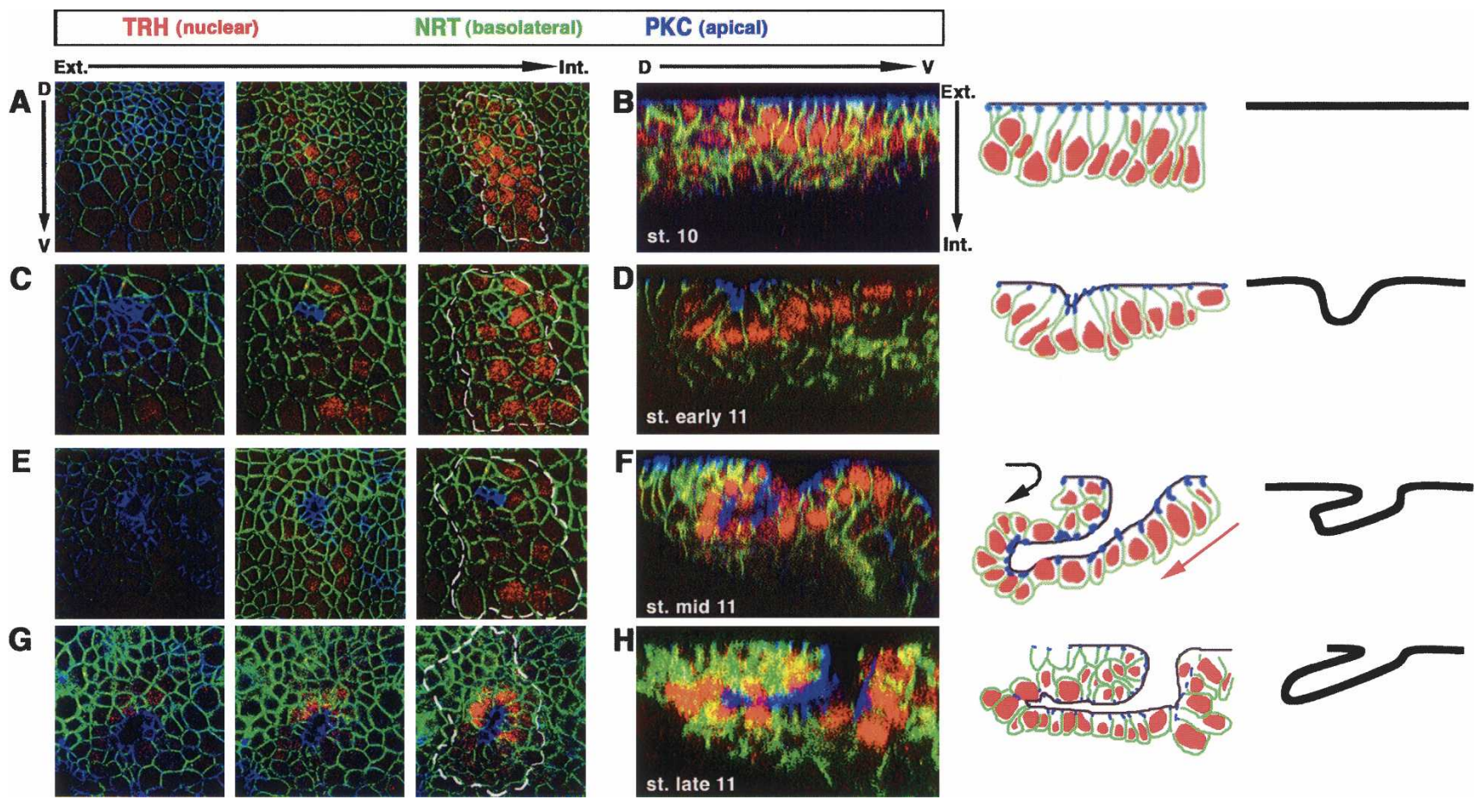

Figure 1. Wild-type sequences of cell shape changes during tracheal invagination. Invagination of a tracheal placode is observed either using consecutive sections from the surface of the epithelium into the placode $(A, C, E, G)$ or a single perpendicular section through the middle of the placode together with schematic representations $(B, D, F, H)$. Here and in subsequent figures, anterior is to the left and dorsal to the top for the consecutive sections. For the perpendicular sections, dorsal is to the left and external to the top. The position of the tracheal placode is marked with a white dashed line. Tracheal cells are labeled using an anti-trachealess antibody (TRH). Anti-Neurotactin (NRT) labels the basolateral and basal sides of all epithelial cells, while PKC labels their apical side. In the schematic diagrams, the dark line delineates the apical surfaces of the cells. $(A, B)$ At stage 10 before invagination, cells form a flat epithelium. $(C, D)$ At the onset of invagination at early stage 11 , a small group of cells has reduced its apical perimeter, and the epithelium begins to bend. Note that the most apical region of those cells lies in a deeper position than the neighboring ones. $(E-H)$ As invagination proceeds during mid- and late stage 11, the apical surface of the invaginating cells is found in an even deeper position. Cells of the dorsal side of the placode have rotated completely around their axis and are found inside the embryo (black arrow), while ventral cells gradually slide beneath (red arrow), both movements leading to the formation of a finger-like structure. 
those of the other tracheal cells (Fig. 1D). By middle stage 11 , the invagination proceeds further as we can now detect the apical marker of the cells in an even deeper position (Fig. 1E). In addition, at this stage we observe a significant change in the invagination behavior of these cells. On the dorsal side, cells begin a rotation-like movement folding to form a new layer of cells below the epidermal surface (Fig. 1F, black arrow). On the ventral side, cells slide below the invaginating dorsal cells (Fig. $1 \mathrm{~F}$, red arrow). As a result, a finger-like structure originates in a process that has evolved from a cell monolayer to a "three-layer organization" (two cell layers initiating a tube below the epidermis layer) (see schemes in Fig. 1). As development proceeds, this finger-like structure elongates dorsally incorporating more tracheal cells from the embryonic surface toward the inside (Fig. 1G,H).

\section{Genetic control of tracheal invagination}

What are the genes and signals responsible for triggering the cell shape changes in tracheal invagination? trh is one of the first genes to be specifically expressed in the cells that will develop as tracheal cells and is responsible for conferring tracheal fate (Isaac and Andrew 1996; Wilk et al. 1996). In trh mutant embryos, there is no tracheal invagination; no local apical constriction takes place and the above-mentioned cell shape changes do not occur (Fig. 2C,D, cf. A,B for a wild type). Thus, all the cell morphological changes in tracheal invagination are induced by the gene expression program triggered by trh. Two targets of trh are the rhomboid (rho) and breathless (btl) genes (Wilk et al. 1996; Llimargas and Casanova 1997); rho codes for a transmembrane protein that specifically cleaves the EGF ligand (Lee et al. 2001) and thus triggers activation of the EGF pathway in the tracheal cells, while btl codes for a homolog of the FGF receptor (Klambt et al. 1992) that enables tracheal cells to respond to clusters of surrounding cells expressing an FGF homo$\log$ (Sutherland et al. 1996).

In rho mutant embryos, tracheal invagination is altered from its beginning at early stage 11: Instead of a few cells initiating invagination at a precise point and being subsequently followed by the remaining cells, a broad domain of the tracheal placode appears to bend in a general way forming a broad cavity (Fig. 2E,F). Cells on the dorsal side do not carry out their rotation movement, and thus a "three-layer organization" is not shaped at this stage (Fig. 2F). Hence, EGFR signaling is responsible for the initiation of local apical constriction and the arrangement of the ensuing cell movements. In the absence of EGFR signaling and as a consequence of the initial aberrant cell movements, some of the cells will remain at the embryonic surface, and consequently an abnormal tracheal tree is formed (Llimargas and Casanova 1999). Conversely, in btl mutants, invagination begins normally until the formation of the finger-like structure, but then this structure does not extend and remains shorter (Fig. 2G,H). These results are consistent with the described role of FGF signaling as a chemoattractant in tracheal cell migration (see Ghabrial et al.
2003). We note that, in the absence of EGFR signaling, although early invagination is impaired, a finger-like structure is eventually formed at later stages (schematic representation in Fig. 2E; data not shown), raising the possibility that, indeed, FGF-signaling-induced migration could also contribute to the formation of this structure. Here, we have confirmed this possibility because in the absence of both EGFR and FGF signaling, invagination initiates as in rho mutants but rotation of dorsal cells does not occur, and a finger-like structure is never formed; instead tracheal cells remain as a cavity (Fig. 2I, J).

sal function shapes tracheal invagination by down-regulating EGFR signaling on the dorsal side of the tracheal placode

Invagination begins always at the same position in all the placodes (Fig. 1C). Tracheal placodes are divided into dorsal and ventral subdomains by the activity of the sal gene, which around stage 10 is expressed in the ectoderm of the central body segments in domains that overlap with the dorsal region of each tracheal placode (Kuhnlein and Schuh 1996). It is at this border between dorsal (sal positive) and ventral (sal negative) subdomains where invagination originates (Fig. 3A). Moreover, sal-expressing cells correspond to those that turn dorsally, while sal-negative cells are the ventral ones sliding underneath (Fig. 3B,C). These observations suggest a central role for sal in organizing tracheal invagination, which is confirmed by the analysis of sal mutants. Two features deviate from the wild-type invagination in sal mutants. First, invagination does not begin at a single point, but instead two invagination sites are apparent in the placode (Fig. 2K). By means of a construct that drives lacZ expression under the control of the sal promoter (Kuhnlein et al. 1997), we have mapped one invagination to the dorsal half of the placode and the other to the ventral half (data not shown). Second, in sal mutants, dorsal tracheal cells do not rotate, which is reminiscent of rho mutant embryos (Fig. 2L). Again similarly to rho embryos, a finger-like structure forms at a later stage (schematic representation in Fig. 2M; data not shown), likely dependent on FGFR signaling.

Due to the similarities between the sal and rho mutant phenotypes, we have examined whether sal could have a role by modulating the activity of the EGFR pathway in the dorsal tracheal cells. Thus, we examined the pattern of EGFR activation using a monoclonal antibody against the active, diphosphorylated form of ERK (Gabay et al. 1997). In the wild-type placode, as invagination proceeds, there is a stronger signal in the ventral part of the placode, indicating a higher activation of the EGFR pathway (Fig. 3D). However, in sal mutants, dpERK staining is stronger dorsally (Fig. 3E), suggesting that sal function is responsible for down-regulation of EGFR activity in the dorsal tracheal cells.

In order to test this possibility and to establish what mechanism could account for this down-regulation, we have analyzed the expression of rho in the tracheal plac- 


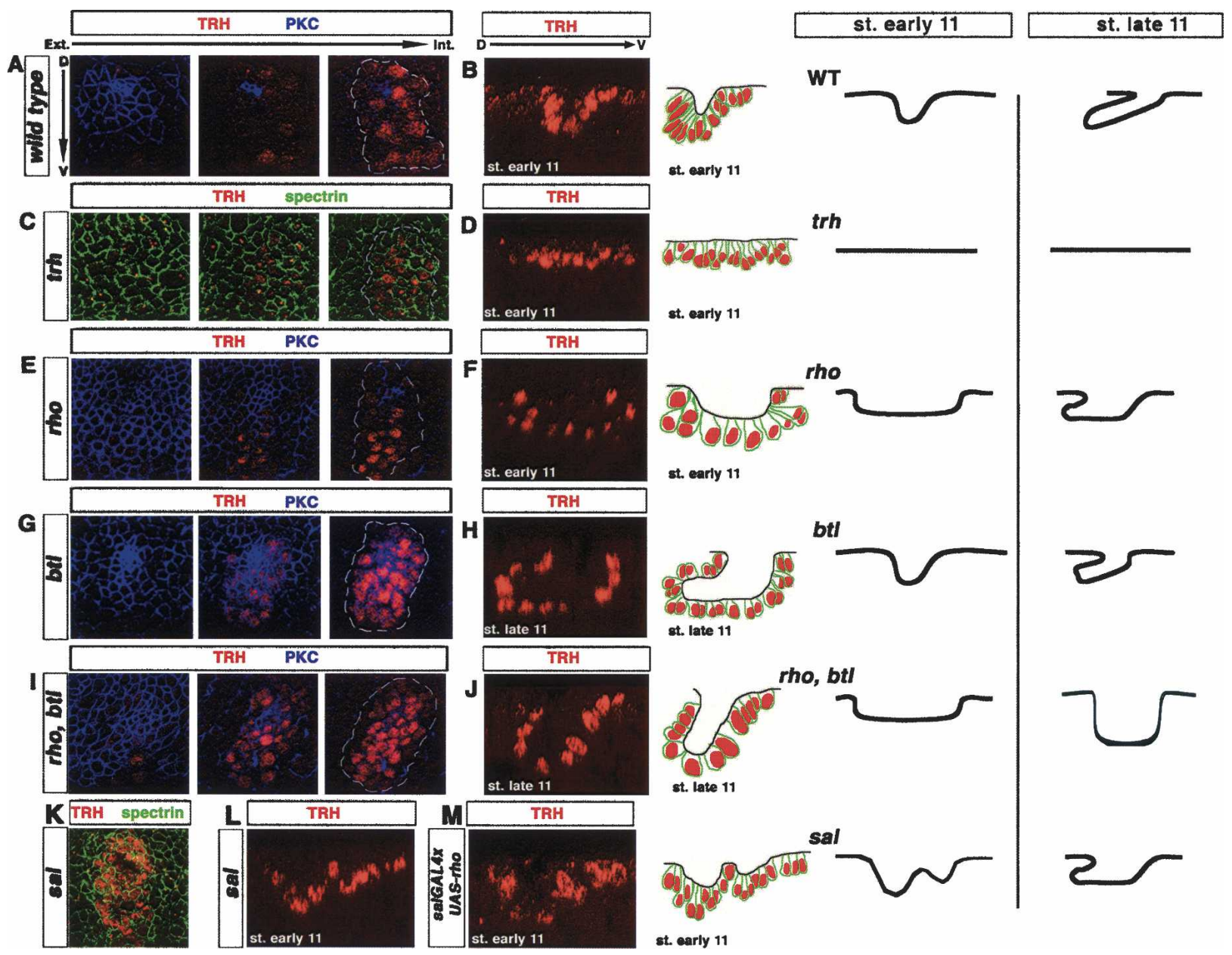

Figure 2. Genetic control of cell shape changes during tracheal invagination. Consecutive confocal sections $(A, C, E, G, I)$ and single perpendicular sections with schematic representation of the apical position of cells at early $(B, D, F, L, M)$ and late $(H, J)$ stage 11 in different mutant backgrounds. Here and in Figure 5, anti- $\alpha$ spectrin is used to label all epithelial cell membranes. $(A, B)$ In wild-type embryos, invagination is initiated with the apical constriction of a small, spatially restricted, group of cells and curving of the epithelial layer. $(C, D)$ In trh mutants, no sign of apical constriction is detected, and the epithelium remains flat. $(E, F)$ In $r h o$ mutants, initiation of apical constriction within a few cells is not observed. Instead, aberrant invagination leads to the formation of a large cavity in the tracheal placode. By the end of stage 11, an abnormal finger-like structure is observed. $(G, H) b t l\left(F G F R^{-}\right)$mutants exhibit localized apical constriction and finger-like formation at the onset of invagination. Nevertheless, the finger does not extend further during stage 11. $(I, J)$ Initiation of invagination in rho, btl placodes is as in rho mutants. However, at late stage 11, a finger-like structure is never observed. $(K, L)$ In sal mutants, invagination is initiated at two positions and ultimately gives rise to an abnormal finger-like structure at late stage 11. (M) Similarly, overexpression of rhomboid driven by salGAL4 in the dorsal half of the placode (see Fig. 3) leads to formation of a double arch at the early stage 11 placode.

ode. At early stage 11 , rho appears evenly distributed in the two halves of the placode (data not shown), but by mid-stage 11, we can see less rho protein on the dorsal side of the placode (Fig. 3F). Moreover, the dorsal downregulation of rho is dependent on sal activity, because it is abolished in sal mutants (Fig. 3G), suggesting that sal modulates EGFR signaling by limiting the amount of rho protein. In addition, overexpression of $r$ ho within dorsal tracheal cells, using a salGAL4 driver, gives rise to an invagination phenotype similar to the one in sal mutants: Two initial invagination sites appear in the placode, and cells from the dorsal side do not rotate (Fig. 2M). This result suggests that down-regulation of EGFR activity, via down-regulation of rho, is a major function of sal in promoting the specific migratory behavior of the dorsal tracheal cells. In sum, these results indicate that EGFR signaling is required to organize the distinct cell movements underlying tracheal invagination, and that it is equally important to have an interphase between cells with higher and lower levels of EGFR signaling.

\section{Myosin localization in tracheal invagination}

In a next step we have investigated what are the cytoskeleton components regulated by the patterning genes and EGFR signaling that trigger the apical constriction at the onset of tracheal invagination. Nonmuscle Myosin II, and in particular its role as an actin-based motor pro- 


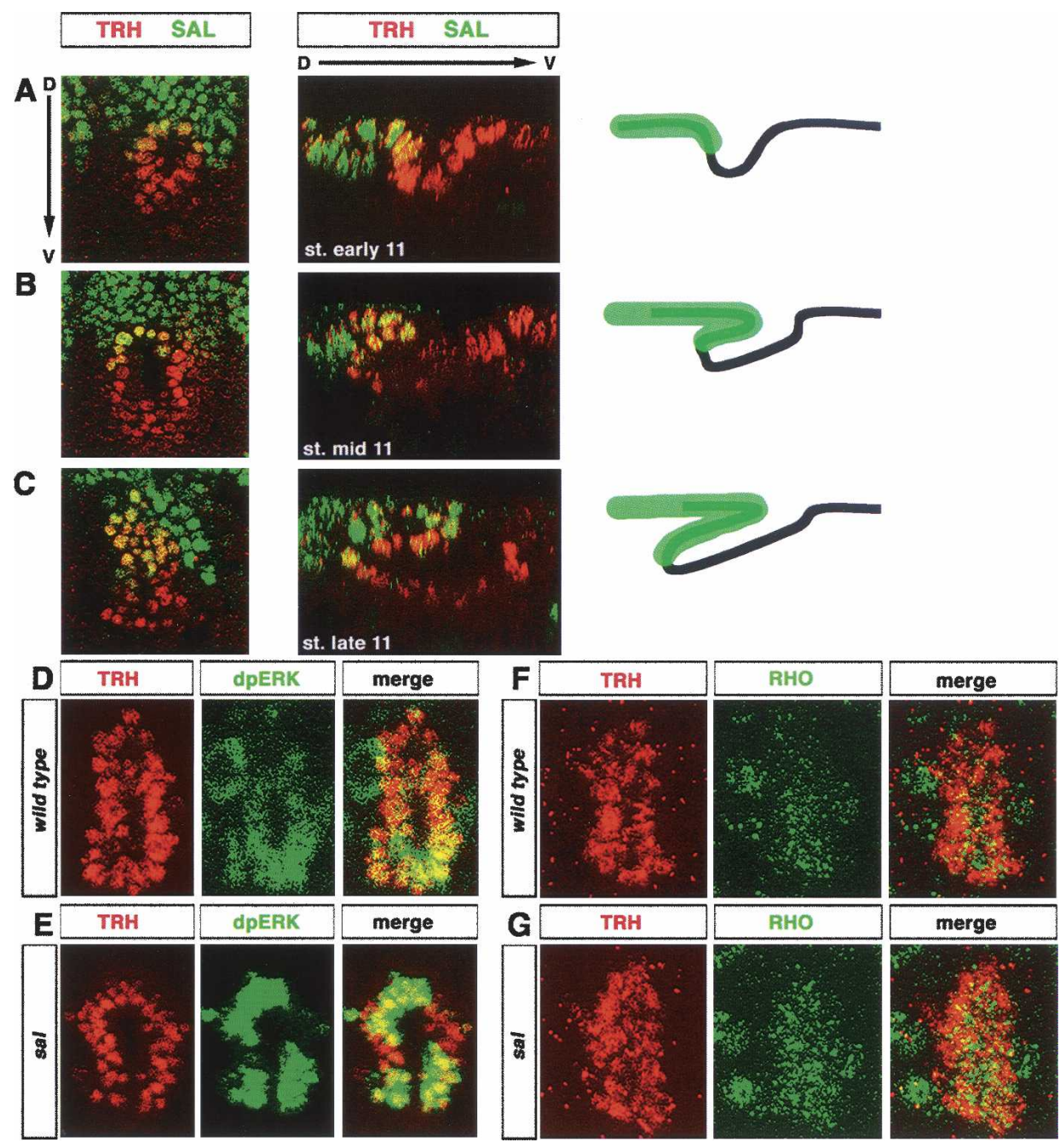

Figure 3. sal down-regulates EGFR activity in the dorsal tracheal cells. $(A-C)$ The tracheal placode is divided into a dorsal, spalt (SAL)-positive domain, and a ventral, SAL-negative one. $(A)$ The invagination point is located at the border between these two domains. $(B, C)$ SAL-positive cells contribute to the formation of the roof of the extending finger. In the schematic diagram, black lines represent the apical surfaces of the tracheal cells, while green represents the domain of SAL expression. $(D, E)$ dpERK is detected at higher levels in the ventral half of the wild-type placode, while the opposite is observed in sal mutants. $(F, G)$ Rhomboid accumulation is higher on the ventral side of wild-type placodes (mean signal amplitude of the ventral side of 93.76 [SD 18.14]; cf. 58.65 [SD 16.69] of the dorsal side). In contrast, Rhomboid is more evenly distributed in sal mutants (mean signal amplitude of the ventral side of 122.17 [SD 24.96]; cf. 115.80 [SD 34.04] of the dorsal side).

viding contractile force, mediates many morphogenetic events such as gastrulation of Drosophila, sea urchin, and Xenopus, and neurulation in vertebrates (for review, see Pilot and Lecuit 2005). Therefore, we have addressed whether Myosin II could drive some of the cell changes underlying tracheal invagination. Myosin II is a hexamer composed of two heavy chains (MHC), two light chains (MLC), and two regulatory light chains (MRLC) (Korn and Hammer 1988). We have used an antibody recognizing the Drosophila MHC subunit, encoded by zipper (zip) (Young et al. 1993), and a GFP-tagged form of the MRLC subunit, encoded by spaghetti squash (sqh) (Karess et al. 1991; Royou et al. 2004), to analyze myosin distribution. We have found that there is a distinct accumulation of myosin at the apical side of the cells at the site of in- vagination, outlining a ring around the edge (Fig. 4A,B). The potential contribution of myosin to tracheal invagination is further stressed by its early apical enrichment in those cells that initiate apical constriction (Fig. 4C,D). Likewise, we have found that actin, which was shown to accumulate at the tracheal cell surfaces facing the invagination hole (Llimargas and Casanova 1999), is also enriched apically in the cells at the site of invagination, although in a broader domain than myosin (Fig. 4A,E). Indeed, double-labeling experiments indicate an overlapping localization of actin and myosin in the apical ring around the invaginating edge (Fig. 4A), suggesting that the interaction of actin and myosin could have an important role in driving the cell movements required for tracheal cell invagination. We have thus addressed 
Figure 4. Actin and myosin II distribution in tracheal cells. (A) Myosin II, as detected with an anti-zipper antibody, is specifically localized at the site of invagination. (B) Myosin II and actin colocalize at the invagination point. $(C)$ Restricted distribution of zipper is seen in a perpendicular section. $(D)$ Accumulation of Myosin II is detected at the onset of invagination in the cells initiating apical constriction (white line with an asterisk), as visualized by a GFP-tagged form of Myosin II light chain (sqh-GFP). (E) In contrast to the restricted zipper distribution, actin is strongly enriched apically in all tracheal cells during invagination. $(F)$ A non-actinbinding YFP-Myosin $\mathrm{II}^{\mathrm{DN}}$ accumulates irregularly at the invagination cells (outlined in yellow) forming noncontinuous apical patches. This mutated version of Myosin II construct is expressed using the GAL4 driver $69 \mathrm{~B}$, and its distribution is followed using an anti-GFP antibody.
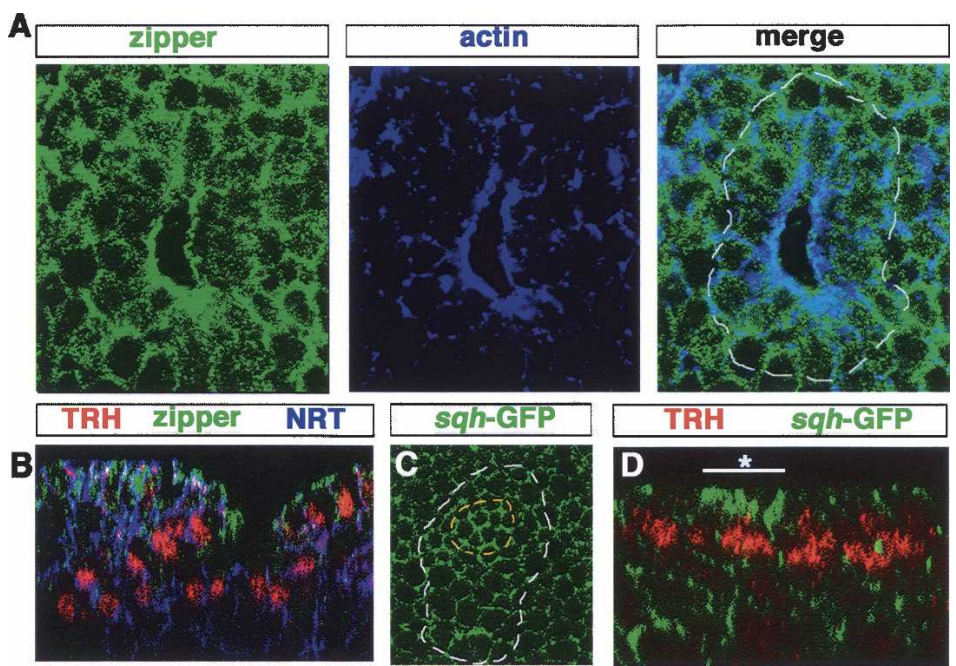

sqh-GFP
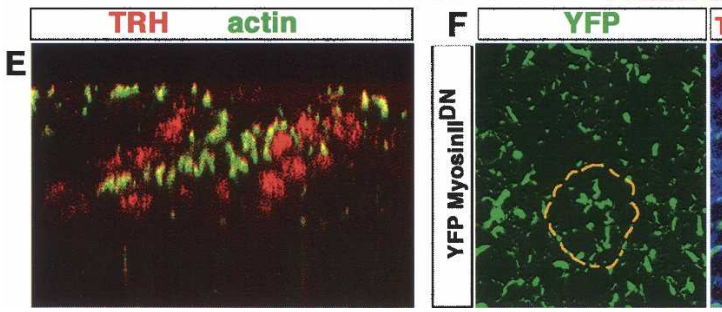

whether myosin localization around the invagination is dependent on its interaction with actin. To do so, we have taken advantage of a recently engineered mutant form of myosin that has its actin-binding motor head replaced by an YFP moiety, although it apparently does not produce a dominant-negative effect, presumably because of high levels of endogenous myosin (DawesHoang et al. 2005). When expressed in the invaginating trachea, this mutated form of myosin is not organized as a distinct and uniform ring around the edge; instead, it shows an uneven distribution and punctuated accumulation (Fig. 4F). This result indicates that, indeed, myosin is properly recruited and organized at the invaginating edge by its association with actin and suggests that contraction of the actin-myosin complex could provide a force driving invagination.

The localization of actin and myosin to the invaginating edge is dependent on the genes we have described here as being required for directing tracheal invagination. In trh mutants, there is no specific accumulation of either actin (Llimargas and Casanova 1999) or myosin, and there is no distinction with the remaining ectodermal cells (Fig. 5, cf. A and B). In the absence of EGFR signaling, neither myosin (Fig. 5C) nor actin (Fig. 5E) accumulates tightly around the invagination edge; instead, they form aggregates, which are more obvious in the case of the actin distribution (Fig. 5E). These results reinforce the idea of an active role of actin-myosin in tracheal invagination. Thus, we have investigated further its contribution by analyzing zip mutants. In these mutant embryos, initiation of invagination is disrupted in a similar way to rho mutants (cf. Figs. 2E,F and 5G,H).
Besides, actin distribution appears modified in zip mutants, because it is not as specifically localized to the apical region and instead extends along the lateral membrane (Fig. 5F), suggesting that the actin-myosin complex becomes more concentrated to the apical side of the invaginating tracheal cells as a result of myosin activity.

cv-c, a Rho-GAP, as a mediator of trhand EGFR-induced invagination

How is the information from trh and the EGFR signaling pathway transmitted into the distribution of the actinmyosin complex that underlies tracheal cell reshaping? A good candidate for a mediator molecule could be the product of the $c V-c$ gene, a RhoGAP that has been shown to have a role in actin remodeling and that is specifically expressed in the tracheal placodes (Fig. 6A,B; Denholm et al. 2005). Interestingly, we have found that $c V-c$ tracheal expression is regulated by both trh and the EGFR pathway: Its expression is completely dependent on trh function (Fig. 6C) and is greatly reduced in mutants impairing EGFR signaling (Fig. 6D). Furthermore, ectopic activation of the EGFR pathway also results in ectopic $C V$ - $c$ expression (Fig. 6E). Altogether, these results suggest that trh regulates $c V$ - $c$ expression by two different mechanisms, one EGFR-dependent and another EGFRindependent (Fig. 7E).

We have investigated whether $c V-c$ function is required for proper tracheal invagination. There is a variably penetrant tracheal phenotype in $\mathrm{CV}-\mathrm{C}$ mutants $174 \%$ of the placodes show an abnormal invagination in $\mathrm{CV}-\mathrm{C}^{7}$ homozygous mutant embryos out of 42 placodes ana- 


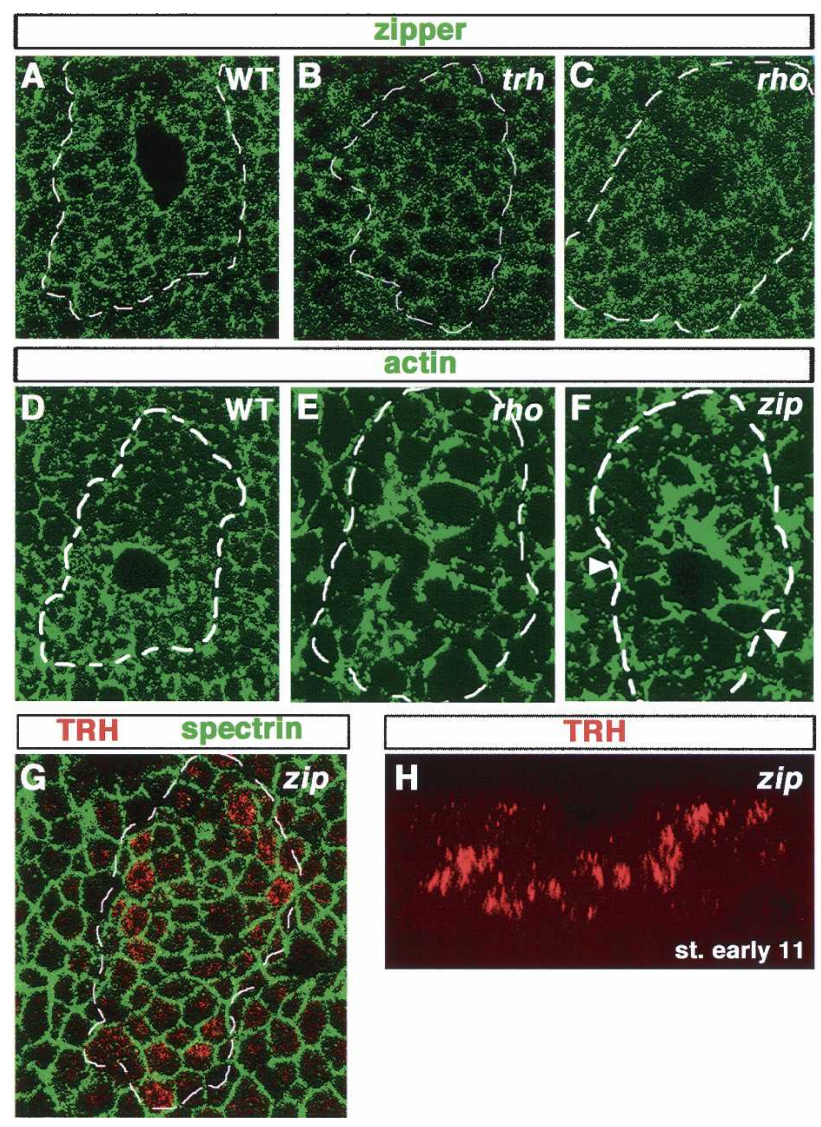

Figure 5. Myosin II accumulation during invagination depends on trh and EGFR signaling. (A) Using sqh-GFP, apical enrichment of Myosin II is detected in a small group of cells in the center of the placode prior to invagination. Note that $s q h$-GFP within this group of cells (outlined in yellow) is stronger and more continuous than in surrounding cells. $(B)$ No Myosin II enrichment is detected in trh placodes visualized using antizipper. Note that the levels are similar inside and outside of the placode, and with the nontracheal cells of a wild-type embryo (as shown in $A$ ). Myosin II $(C)$ and apical actin $(E)$ enrichment are strongly affected in rho placodes (cf. with wild type in Fig. 4B). Wild-type $(D)$ and zip $(F)$ tracheal placodes. In contrast to the distinct apical enrichment of actin seen in wild type $(D)$, in a zip tracheal placode, actin is evenly distributed along the entire cell surface (e.g., see arrowheads in $F) .(G, H)$ In zip mutants, the small group of cells that initiates apical constriction is not observed, and tracheal cells form a large cavity during invagination.

lyzed). Such variability has been reported for other features of the null $c V-c$ mutant phenotype (Denholm et al. 2005). Invagination in $c V-c$ mutants is reminiscent to what is observed in the absence of EGFR signaling and in zip mutants; there is no proper apical constriction (Fig. 6F), dorsal cells do not rotate, but instead the tracheal cells remain in a broad cavity (Fig. 6G,H). Moreover, in $c V-c$ mutants myosin distribution is severely disrupted: Instead of a well-shaped and uniform ring around the edge of the invagination site, there is a patchy distribution with the formation of myosin aggregates (Fig. 6I). Actin distribution is also impaired in the tra- cheal cells of $c V-c$ mutants, as the regional differences inside the cells are disturbed: Apical levels appear to be lower, and we detect actin at the basal membrane, as opposed to the apical wild-type situation. Additionally, the remaining apically located actin appears abnormal and seems to form similar aggregates (Fig. 6J) that colocalize with myosin (Fig. 6I).

Given that $c v-c$ expression is regulated by EGFR signaling, the similarities between $r h o, c v-c$, and zip phenotypes and the abnormal distribution of myosin in rho and $c V-c$ mutants, it is likely that EGFR activity on tracheal invagination is mediated at least in part by $\mathrm{CV}-\mathrm{C}$ function on actin-myosin. It should be noted that the pattern of actin-myosin accumulation appears to be more strongly disrupted in $\mathrm{CV}-\mathrm{c}$ mutants (Fig. 6J) than in the absence of EGFR signaling (Fig. 5C,E), which is compatible with the observation that there is some residual $C V-C$ expression in the absence of EFGR signaling (Fig. 6D). To further assess a functional link between the roles of the EGFR pathway and $c v-c$ on invagination, we have investigated whether we could find a genetic interaction between mutants in these different components. To this end, we have used $s p i^{2}$, a mutation in the EGFR ligand spitz that gives rise to a mild invagination phenotype in homozygous embryos $135 \%$ of the placodes show an abnormal invagination; $n=29$ ). This phenotype was significantly increased by the presence of a single copy of the $c V-c^{7}$ mutation $167 \%$ of the placodes show an abnormal invagination; $n=39$ ). Altogether, these results point to the RhoGAP $c V-c$ as a key intermediate between patterning and signaling pathways and actin-myosin function during tracheal cell invagination.

\section{Rho1 is a likely substrate of $\mathrm{cV}-\mathrm{c}$ during tracheal invagination}

RhoGAPs act by promoting the transition of RhoGTPases from their GTP-bound active state to a GDP-bound inactive state (Symons and Settleman 2000). Previous results have shown that $\mathrm{cv}-\mathrm{c}$ can interact with different RhoGTPases in a tissue-specific manner (Denholm et al. 2005). To identify the RhoGTPase acting as a substrate for $\mathrm{cv}-\mathrm{c}$ in tracheal invagination, we have analyzed whether mutants for different Drosophila RhoGTPases could also have a defective tracheal invagination. We have found that this was the case for Rho1-null mutant embryos (Fig. 6K). Conversely, homozygous embryos for a chromosome carrying mutant alleles for three other RhoGTPases (Rac1, Rac2, and Mt1) displayed normal tracheal invagination, although tracheal development was impaired at later stages (Lee and Kolodziej 2002; data not shown). Confirming the role of Rhol as a substrate of $\mathrm{cv}-\mathrm{c}$ in tracheal invagination, we observed a genetic interaction between hypomorphic mutant alleles for these genes. Specifically, since a $c V-c$ mutant is presumed to cause an increase in the activity of its substrate, we would expect that a reduction of $\mathrm{CV}-\mathrm{c}$ activity would ameliorate the phenotype of a Rho1 allele. Accordingly, the $c V-c^{M 62}$ mutant partially rescued the phenotype of the $R h o 1^{72 R}$ allele (Fig. 6O-R). 
Figure 6. The RhoGAP encoded by $c V-c$ is a mediator of trh-and EGFR-induced invagination and interacts genetically with the small Rho-GTPase Rhol. $(A-E)$ Expression of $c V-c$ mRNA in wild-type $(A, B)$, trh $(C)$, and rho $(D)$ placodes. At stage 11, $\mathrm{CV}$ - $\mathrm{C}$ expression is restricted to the tracheal cells $(A)$ and seems enriched apically $(B)$. No expression is detected in trh mutants, while a low level remains in rho mutants. $(E)$ Overexpression of rhomboid driven in stripes by ptcGAL4 leads to ectopic cv-c expression. Black lines correspond to the limits of the ptcGAL4 expression domain. $(F-H)$ In $c V-c$ placodes, the small group of cells initiating apical constriction is not detected. At early stage 11, formation of a large cavity in the tracheal placode is observed, and by the end of stage 11 , a disorganized finger-like structure is observed. $(I, J)$ In $c V$ - $c$ mutants, actin and zipper colocalize in patches. In addition, actin is now detected along the basolateral and basal sides (e.g., see arrowheads in $J)$. $(K-N)$ In Rho1-null mutants, initiation of apical constriction within a few cells is not observed $(K)$. $(L)$ Instead, aberrant invagination leads to the formation of a large cavity in the tracheal placode. In addition, actin $(M)$ and myosin $(N)$ distributions are strongly affected. $(O, P)$ In a $R h o 1^{72 R}$ hypomorphic mutant background, localized apical constriction is not detected $(O)$, and invagination proceeds, forming a large cavity $(P) . \quad(Q, R)$ In double homozygous $R h o 1^{72 R}$; $C V C^{M 62}$ mutant placodes, initiation of apical constriction and finger-like formation are rescued at the onset of invagination. Compare the degree of apical cell constriction using PKC marker between single $R h o 1^{72 R}$ mutant $(P)$ and double mutant $(R)$ (white arrows).
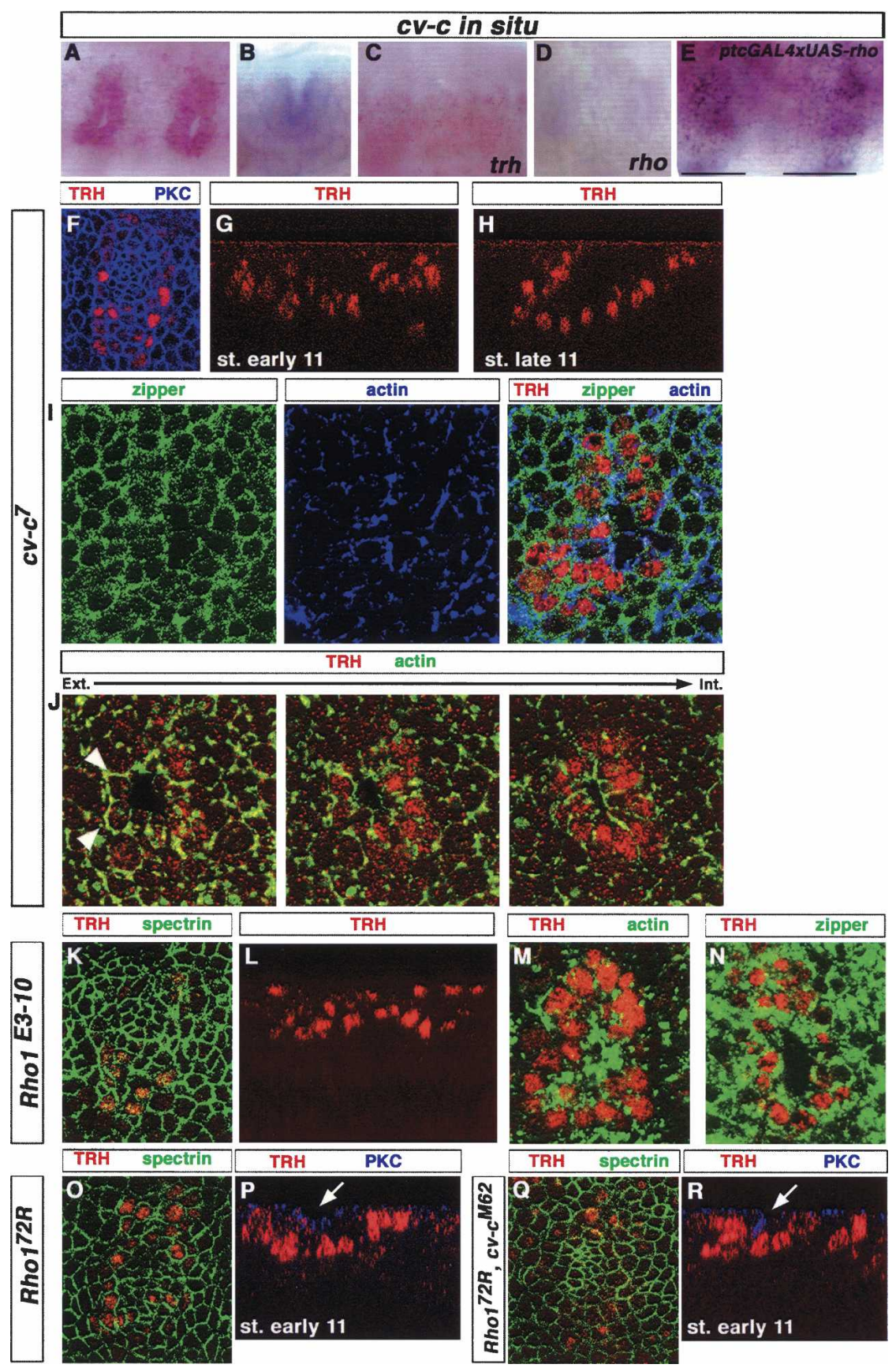

cv-c partially rescues apical actin organization in tracheal invagination of Egfr mutants

As mentioned before, $c V-c$ activity is required for the distinct apical accumulation of actin in the invaginating cells. At present, the mechanism of $c v-c$ activity is not known, although it has been suggested that $\mathrm{cv}-\mathrm{c}$ protein could localize to the membrane, where it would regulate an interaction between the plasma membrane and the actin cytoskeleton (Denholm et al. 2005). Confirmation of this hypothesis will have to wait for the availability of an anti-cv-c antibody. Yet, we have observed that the $c V-c$ RNA appears highly enriched in the apical side of the invaginating tracheal cells (Fig. 6B), although at present we cannot state whether this distribution is functionally significant.

To further assess the potential role of $c V-c$ in terms of actin localization and cell invagination, we have ubiquitously expressed $c V-c$ in the ectoderm and analyzed its effects. Under these circumstances we see an increase in actin apical localization in ectodermal cells, showing that expression of $c V-c$ is able to affect actin distribution (Fig. 7A,B). In these embryos, we also observe that some positions that show an increase in apical actin localization are coupled with small patches of cells that appear to have invaginated, since we detect their apical cell sur- 

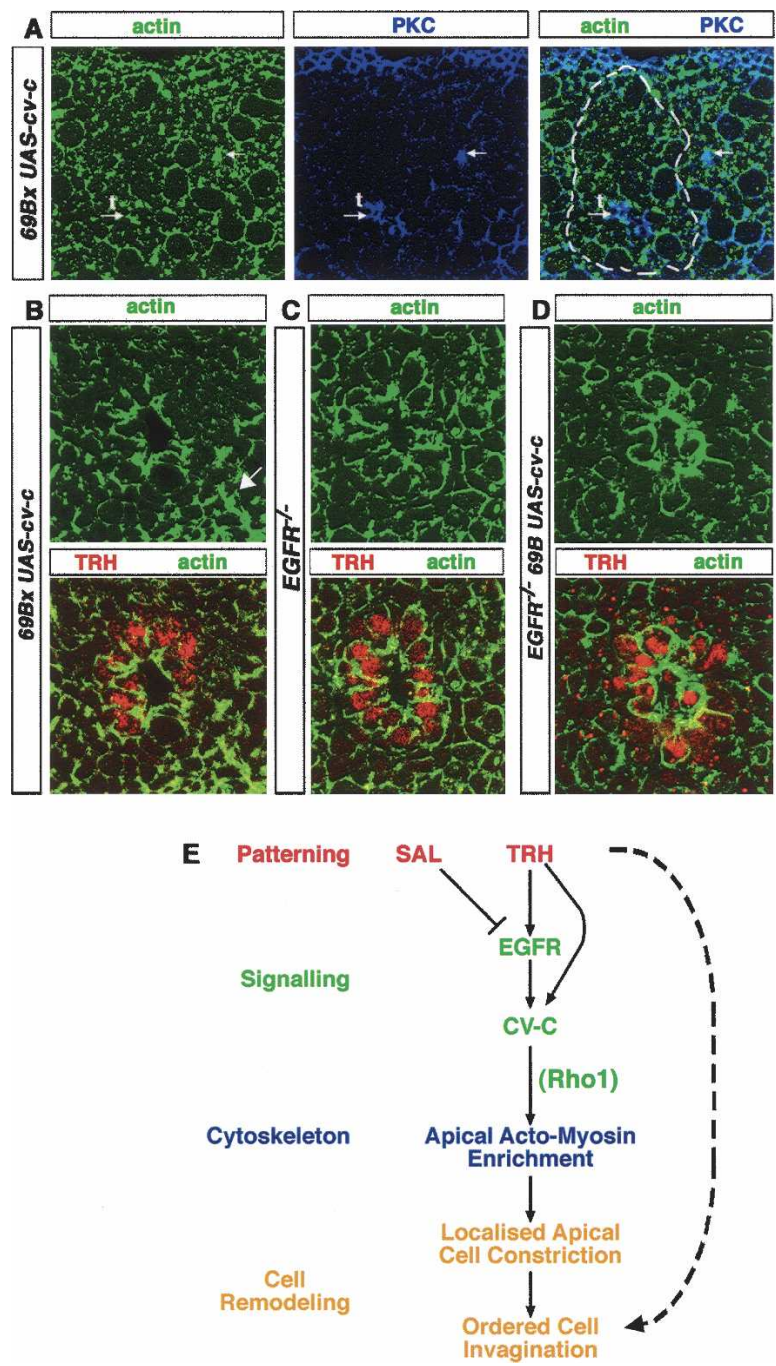

Figure 7. $c V-c$ controls actin distribution. $(A) c V-c$ overexpression driven by 69B directs ectopic invagination of small patches of cells (white arrows) outside the tracheal placode (white arrow with t). The apical cell membranes, visualized by PKC, are detected in more internal sections and colocalize with higher actin levels. (B) Overexpression of $c v-c$ using a 69B driver leads to large actin enrichment in many ectodermal cells, mostly apically (i.e., those marked with an arrow). $(C)$ In an Egfr mutant placode, apical actin accumulation is severely disrupted. (D) In contrast, overexpression of $c V-c$ in this mutant background partially rescues apical actin enrichment. (E) Model for ordered tracheal cell invagination (see text for details).

face, visualized by PKC, deeper inside (Fig. 7A). As mentioned above, loss of EGFR signaling results in decrease of apical actin accumulation (Fig. 5C) and invagination defects (Fig. 2E,F). It is likely that effectors other than $\mathrm{cV}-\mathrm{c}$ act downstream of the EGFR pathway to organize the cytoskeletal modifications required for tracheal invagination. Thus, we would not expect a complete rescue of the invagination defects seen in mutants for the EGFR pathway by $c V-c$ expression alone. However, since we have found that $c V-c$ activity is sufficient to affect actin organization, we have examined whether expres- sion of $c V-c$ could at least partially rescue actin accumulation defects in tracheal invagination of EGFR pathway mutants. In mutants for Egfr ${ }^{f 2}$, an amorphic mutation with a strong tracheal invagination phenotype, the tight accumulation of actin around the invagination edge is disrupted (Fig. 7C). However, ubiquitous expression of $c V-c$ is able to partially restore the distinct apical actin accumulation (Fig. 7D). Altogether, the above results illustrate the potential of $c \mathrm{~V}-\mathrm{c}$ activity in apical actin accumulation in tracheal cell invagination.

\section{Discussion}

\section{Setting up an invagination field and organizing invagination progression}

Our results suggest a two-step model by which trh induces and organizes tracheal invagination. First, trh activity appears to outline an invagination field, a region of cells that acquire the competence to invaginate. This effect can be clearly observed in mutants that impair EGFR signaling; in those embryos, trh activity is still able to promote a broad depression of the trh-expressing cells that will only further reorganize due to their ability to migrate in response to FGFR signaling. In this regard, there are clearly some consequences of trh that are independent of EGFR signaling and could be connected with the potential of trh to induce a general depression. For instance, we have found that the microtubule network is highly enriched and polarized apically at the site of invagination; while this arrangement is absent in trh mutants, it remains present in the abnormal invaginating tracheal placodes in the absence of both FGF and EGFR signaling (data not shown).

A second outcome of trh is accomplished by the triggering of EGFR signaling, which leads to the spatial and temporal organization of tracheal invagination. It is the activity of the EGFR pathway that converts the tracheal cell potential to invaginate into the organized process, resulting in a "three-layer organization" and initiation of tube formation. A partner required for the organization of tracheal invagination is sal, which is expressed in the dorsal half of the tracheal placode and is responsible for the different morphology and behavior of the cells between the two sides of the placodes. We have shown that the role of sal is, at least in part, achieved through downregulation of EGFR signaling activity. However, it is not clear how this modulation is translated into differences in invaginating behavior. For example, we have not been able to detect differences in level or distribution of cytoskeletal components along the sal expression border. An intriguing possibility would be that down-regulation of EGFR signaling gives rise to cells with different forces or stiffness (perhaps due to different levels of actin-myosin contractility), and the resulting apposition of two invaginating cell populations with different properties could force one of them to fold and initiate dorsal-oriented rotation, while the other would slide down under the former.

It is worth noting that a well-organized invagination is 
an absolute requirement for tracheal morphogenesis. All the mutants that cause an abnormal invagination give rise to an impaired tracheal system in which some branches do not develop or develop deficiently. Thus, for example, rho mutants, which were originally thought to affect specifically the formation of two branches, have a general defect in invagination, and many tracheal cells remain clustered at the embryonic surface (Llimargas and Casanova 1999). In this regard, an important outcome of proper tracheal invagination appears to be that the tracheal cells reach the appropriate position with respect to the cues that will direct their subsequent migration. As we suggested before, the wild-type organization of the tracheal tree depends on having the appropriate number of cells at the correct position facing those signals, such that a specific number of cells contributes to the formation of the different branches (Llimargas and Casanova 1999).

\section{Cell fate specification and actin-myosin regulation in morphogenesis}

In many cases, cell fate commitment leads to cell shape modifications and rearrangements. Our results here depict a developmental pathway (Fig. 7E) that is initiated by the activity of a gene specifying cell fate $(t r h)$, which triggers a cell signaling pathway (EGFR) that, in turn, organizes cell invagination. A key step in this pathway is the transcriptional activation of a gene coding for a RhoGAP enzyme, $c V-c$, that affects actin-myosin apical distribution, likely by regulation of Rhol activity.

Regulation of RhoGTPases, either by RhoGAPs or RhoGEFs, appears to be a common trait in the control of morphogenesis. Indeed, RhoGAPs and RhoGEFs have been shown to act in different manners to affect actin and myosin. In this regard, some parallelisms can be found between tracheal cell invagination and other morphogenetic events such as gastrulation and neurulation (Pilot and Lecuit 2005). In particular, clear similarities can be seen with the mechanism of myosin regulation in Drosophila gastrulation. In this case, it is also the activity of a patterning gene (twist) that gives rise to the expression of a signaling molecule (folded gastrulation) that is thought to elicit a signaling pathway requiring a G-protein $\alpha$ subunit (concertina) and a RhoGEF (RhoGEF2). Then, RhoGEF2 ultimately leads to phosphorylation of myosin, which then activates actin binding by myosin and increases actomyosin contractility (DawesHoang et al. 2005). However, in tracheal invagination, the remaining colocalization of myosin and actin in $\mathrm{CV}-\mathrm{C}$ mutants suggest that $c V-c$ is not necessary for the interaction between actin and myosin but instead for the proper localization of the actin-myosin complex. This observation fits well with a recent report that indicates that the $c V-c$ RhoGAP acts on the actin apical accumulation in Malpighian tube morphogenesis and during epithelial dorsal closure (Denholm et al. 2005).

Different RhoGTPases act as substrates of the cv-c RhoGAP enzyme in different tissues (Denholm et al. 2005). Our results indicate that Rhol is the substrate for $\mathrm{cV}-\mathrm{c}$ in tracheal invagination. Notably, there appear to be more RhoGAPs and RhoGEFs molecules than RhoGTPases, which has been interpreted as an indication of the importance of a precise regulation of the transition between active and inactive states of RhoGTPases for different cell processes (see Bernards 2003). Additionally, the fact that mutants for $c V-c$, a negative regulator of Rhol activity, and Rho1 both impair actin apical organization and cell invagination in the tracheal placodes illustrates the importance of an appropriate regulation of RhoGTPase activity to achieve proper actin organization and cell behavior. In this regard, the fact that the $\mathrm{CV}-\mathrm{C}$ RhoGAP has a pivotal role in tracheal invagination does not rule out that additional regulatory mechanisms that act on RhoGTPases could also be in place in tracheal invagination. The variable penetrance of null $c \mathrm{~V}-\mathrm{c}$ RhoGAP phenotypes suggests the possible existence of other invagination-regulating molecules under the control of trh. Additionally, EGFR signaling is only one of the programs elicited by the activity of trh. Altogether, these observations indicate that the developmental pathway that induces and organizes tracheal invagination must have diverse branches with additional target outcomes. We suggest that many morphogenetic events share the same basic operational logic; leading from patterning genes and cell signaling pathways to cell shape changes, although each case may involve diverse target molecules acting at different steps in the regulation of the actin-myosin complex.

\section{Materials and methods}

\section{Fly strains}

Description of most genetic elements can be found at http:// flybase.net (FlyBase Consortium1999). The following loss-offunction mutations were used: $\operatorname{trh}^{1}, b t I^{L G 19}, z i p^{1}, c v-c^{7}, c V-c^{M 62}$, Rho1 ${ }^{E 3.10}$, Rho1 $^{72 R}, \operatorname{Rac}^{11}, \operatorname{Rac}^{\Delta}, \mathrm{Mtl}^{\Delta}, \mathrm{spi}^{2}, \mathrm{Egfr}^{\mathrm{f2}}, \mathrm{rho}^{7 \mathrm{~T} 3}$ (rhomoid-1 rhomboid-3 double mutants) (Wasserman et al. 2000), $D f(5)$, which removes both sal and sal-related (Barrio et al. 1999). CyO-hb-lacZ, CyO-wg-lacZ, TM3-ftz-lacZ, or TM6$U b x$-lac $Z$ blue balancers were used to identify homozygous embryos. The sqh-GFP has been described in Royou et al. (2004). We used the GAL4 system (Brand and Perrimon 1993) for misexpression experiments. The salGAL4 (Calleja et al. 1996; Boube et al. 2000), 69B (Brand and Perrimon 1993; Castelli-Gair et al. 1994), and ptcGAL4 (Speicher et al. 1994) lines were used in combination with UASrho (de Celis et al. 1997), UASYFPMyosinIIDN (Dawes-Hoang et al. 2005), or UAScv-c (Denholm et al. 2005). Crosses with GAL4 lines were done at $29^{\circ} \mathrm{C}$, except for those with UASCV-c, which were performed both at $18^{\circ} \mathrm{C}$ and at $29^{\circ} \mathrm{C}$. In this case, we observed a stronger effect on actin localization at $29^{\circ} \mathrm{C}$ than at $18^{\circ} \mathrm{C}$.

\section{Immunohistochemistry}

Embryos were staged according to Campos-Ortega and Hartenstein (1985) and stained according to standard protocols. Immunostaining was performed on embryos fixed in $4 \%$ formaldehyde for $20 \mathrm{~min}$, except for anti-rho staining, in which embryos were fixed for $10 \mathrm{~min}$; anti-dpERK staining, in which embryos were fixed for $20 \mathrm{~min}$ in $8 \%$ formaldehyde; and anti-zipper as described in Bloor and Kiehart (2001). 
Photographs were taken using a Normarski optic in a Nikon Eclipse 80i microscope. All fluorescent images were collected using confocal microscopy (Leica TCS-SP2-AOBS system, Leica DM IRE2 microscope and LCS software) with a pinhole of 1 . They are all single sections except Figure 3D-G, which shows projections of several confocal sections.

RNA in situ hybridization used a $c V$ - $c$ probe, as described previously (Denholm et al. 2005).

We used the following primary antibodies: rabbit anti-GFP (1:1000; Molecular Probes), mouse and rabbit anti-ßgal (1:1000; Promega and Cappel, respectively), mouse anti-spectrin and anti-Nrt (1:5; DSHB), anti-mouse dpERK (1:50; Sigma), mouse anti-actin (1:1500; MP Biomedicals), rabbit anti-PKC (1:500; Santa Cruz Biotechnology), anti-Rho (1:2000) (Sturtevant et al. $1996)$ at 1:2000, rabbit anti-Sal (1:1000; gift from A. Salzberg), rabbit anti-zipper (clone 656, 1:500) (Kiehart and Feghali 1986), and rabbit and rat anti-Trh (1:10 and 1:400, respectively; our own stocks, made by N. Martín). Biotinylated or Cy2, Cy3, and Cy5 secondary antibodies (Jackson ImmunoResearch) were used at a dilution of 1:400.

\section{Acknowledgments}

We thank A. Brand, M. Calleja, G. Morata, M. Freeman, D. Kiehart, A. Salzberg, the Bloomington Stock Center, and especially J.Castelli-Gair for providing flies and materials; S. Araujo, O. Grimm, A. Guichet, M. Milán, M. Llimargas, and in particular D. Shaye for critically reading the manuscript; and M. Llimargas for discussions. We also thank N. Martín and R. Méndez for their technical assistance and Lídia Bardia for the confocal microscopy support and advice. V.B. was supported by an EMBO long-term fellowship and is now supported by an I3P contract from the Consejo Superior de Investigaciones Científicas (CSIC). This work has been carried out in the framework of the Centre de Referència en Biotecnologia de la Generalitat de Catalunya and supported by the Spanish Ministerio de Educación y Ciencia.

\section{Note added in proof}

After completion of this work, Simões et al. (2006) described the role of $\mathrm{cV}-\mathrm{c}$ in the morphogenesis of the posterior spiracles. Their findings are complementary to those of the present study.

\section{References}

Barrio, R., de Celis, J.F., Bolshakov, S., and Kafatos, F.C. 1999. Identification of regulatory regions driving the expression of the Drosophila spalt complex at different developmental stages. Dev. Biol. 215: 33-47.

Bernards, A. 2003. GAPs galore! A survey of putative Ras superfamily GTPase activating proteins in man and Drosophila. Biochim. Biophys. Acta 1603: 47-82.

Bloor, J.W. and Kiehart, D.P. 2001. zipper Nonmuscle myosin-II functions downstream of PS2 integrin in Drosophila myogenesis and is necessary for myofibril formation. Dev. Biol. 239: 215-228.

Boube, M., Llimargas, M., and Casanova, J. 2000. Cross-regulatory interactions among tracheal genes support a co-operative model for the induction of tracheal fates in the Drosophila embryo. Mech. Dev. 91: 271-278.

Boube, M., Martin-Bermudo, M.D., Brown, N.H., and Casanova, J. 2001. Specific tracheal migration is mediated by complementary expression of cell surface proteins. Genes \& Dev.
15: 1554-1562.

Brand, A.H. and Perrimon, N. 1993. Targeted gene expression as a means of altering cell fates and generating dominant phenotypes. Development 118: 401-415.

Calleja, M., Moreno, E., Pelaz, S., and Morata, G. 1996. Visualization of gene expression in living adult Drosophila. Science 274: 252-255.

Campos-Ortega, A.J. and Hartenstein, V. 1985. The embryonic development of Drosophila melanogaster. Springer-Verlag, New York.

Castelli-Gair, J., Greig, S., Micklem, G., and Akam, M. 1994. Dissecting the temporal requirements for homeotic gene function. Development 120: 1983-1995.

Chihara, T. and Hayashi, S. 2000. Control of tracheal tubulogenesis by Wingless signaling. Development 127: 4433-4442.

Dawes-Hoang, R.E., Parmar, K.M., Christiansen, A.E., Phelps, C.B., Brand, A.H., and Wieschaus, E.F. 2005. Folded gastrulation, cell shape change and the control of myosin localization. Development 132: 4165-4178.

de Celis, J.F., Bray, S., and Garcia-Bellido, A. 1997. Notch signalling regulates veinlet expression and establishes boundaries between veins and interveins in the Drosophila wing. Development 124: 1919-1928.

Denholm, B., Brown, S., Ray, R.P., Ruiz-Gomez, M., Skaer, H., and Hombria, J.C. 2005. crossveinless-c is a RhoGAP required for actin reorganisation during morphogenesis. Development 132: 2389-2400.

FlyBase Consortium. 1999. The FlyBase database of the Drosophila Genome Projects and community literature. Nucleic Acids Res. 27: 85-88.

Franch-Marro, X. and Casanova, J. 2000. The alternative migratory pathways of the Drosophila tracheal cells are associated with distinct subsets of mesodermal cells. Dev. Biol. 227: 80-90.

Gabay, L., Seger, R., and Shilo, B.Z. 1997. In situ activation pattern of Drosophila EGF receptor pathway during development. Science 277: 1103-1106.

Ghabrial, A., Luschnig, S., Metzstein, M.M., and Krasnow, M.A. 2003. Branching morphogenesis of the Drosophila tracheal system. Annu. Rev. Cell Dev. Biol. 19: 623-647.

Isaac, D.D. and Andrew, D.J. 1996. Tubulogenesis in Drosophila: A requirement for the trachealess gene product. Genes \& Dev. 10: 103-117.

Karess, R.E., Chang, X.J., Edwards, K.A., Kulkarni, S., Aguilera, I., and Kiehart, D.P. 1991. The regulatory light chain of nonmuscle myosin is encoded by spaghetti-squash, a gene required for cytokinesis in Drosophila. Cell 65: 1177-1189.

Kiehart, D.P. and Feghali, R. 1986. Cytoplasmic myosin from Drosophila melanogaster. J. Cell Biol. 103: 1517-1525.

Klambt, C., Glazer, L., and Shilo, B.Z. 1992. breathless, a Drosophila FGF receptor homolog, is essential for migration of tracheal and specific midline glial cells. Genes \& Dev. 6: 1668-1678.

Korn, E.D. and Hammer III, J.A. 1988. Myosins of nonmuscle cells. Annu. Rev. Biophys. Biophys. Chem. 17: 23-45.

Kuhnlein, R.P. and Schuh, R. 1996. Dual function of the regionspecific homeotic gene spalt during Drosophila tracheal system development. Development 122: 2215-2223.

Kuhnlein, R.P., Bronner, G., Taubert, H., and Schuh, R. 1997. Regulation of Drosophila spalt gene expression. Mech. Dev. 66: $107-118$.

Lee, S. and Kolodziej, P.A. 2002. The plakin Short Stop and the RhoA GTPase are required for E-cadherin-dependent apical surface remodeling during tracheal tube fusion. Development 129: 1509-1520. 
Lee, J.R., Urban, S., Garvey, C.F., and Freeman, M. 2001. Regulated intracellular ligand transport and proteolysis control EGF signal activation in Drosophila. Cell 107: 161-171.

Llimargas, M. 2000. Wingless and its signalling pathway have common and separable functions during tracheal development. Development 127: 4407-4417.

Llimargas, M. and Casanova, J. 1997. ventral veinless, a POU domain transcription factor, regulates different transduction pathways required for tracheal branching in Drosophila. Development 124: 3273-3281.

-. 1999. EGF signalling regulates cell invagination as well as cell migration during formation of tracheal system in Drosophila. Dev. Genes Evol. 209: 174-179.

Manning, G. and Krasnow, M.A. 1993. Development of the Drosophila tracheal system. Cold Spring Harbor Laboratory Press, Cold Spring Harbor, NY.

Pilot, F. and Lecuit, T. 2005. Compartmentalized morphogenesis in epithelia: From cell to tissue shape. Dev. Dyn. 232: 685-694.

Royou, A., Field, C., Sisson, J.C., Sullivan, W., and Karess, R. 2004. Reassessing the role and dynamics of nonmuscle myosin II during furrow formation in early Drosophila embryos. Mol. Biol. Cell 15: 838-850.

Simões, S., Denholm, B., Sotillos, S., Martin, P., Skaer, H., Castelli-Gair, J., and Jacinto, A. 2006. Compartmentalization of Rho regulators directs cell invagination during tissue morphogenesis. Development (in press).

Speicher, S.A., Thomas, U., Hinz, U., and Knust, E. 1994. The Serrate locus of Drosophila and its role in morphogenesis of the wing imaginal discs: Control of cell proliferation. Development 120: 535-544.

Sturtevant, M.A., Roark, M., O'Neill, J.W., Biehs, B., Colley, N., and Bier, E. 1996. The Drosophila rhomboid protein is concentrated in patches at the apical cell surface. Dev. Biol. 174: 298-309.

Sutherland, D., Samakovlis, C., and Krasnow, M.A. 1996. branchless encodes a Drosophila FGF homolog that controls tracheal cell migration and the pattern of branching. Cell 87: 1091-1101.

Symons, M. and Settleman, J. 2000. Rho family GTPases: More than simple switches. Trends Cell Biol. 10: 415-419.

Vincent, S., Ruberte, E., Grieder, N.C., Chen, C.K., Haerry, T., Schuh, R., and Affolter, M. 1997. DPP controls tracheal cell migration along the dorsoventral body axis of the Drosophila embryo. Development 124: 2741-2750.

Wappner, P., Gabay, L., and Shilo, B.Z. 1997. Interactions between the EGF receptor and DPP pathways establish distinct cell fates in the tracheal placodes. Development 124: 47074716.

Wasserman, J.D., Urban, S., and Freeman, M. 2000. A family of rhomboid-like genes: Drosophila rhomboid-1 and roughoid/ rhomboid-3 cooperate to activate EGF receptor signaling. Genes \& Dev. 14: 1651-1663.

Wilk, R., Weizman, I., and Shilo, B.Z. 1996. trachealess encodes a bHLH-PAS protein that is an inducer of tracheal cell fates in Drosophila. Genes \& Dev. 10: 93-102.

Young, P.E., Richman, A.M., Ketchum, A.S., and Kiehart, D.P. 1993. Morphogenesis in Drosophila requires nonmuscle myosin heavy chain function. Genes \& Dev. 7: 29-41. 


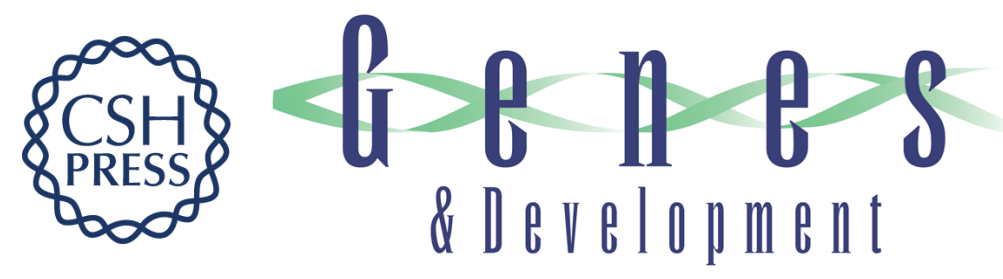

\section{The RhoGAP crossveinless-c links trachealess and EGFR signaling to cell shape remodeling in Drosophila tracheal invagination}

Véronique Brodu and Jordi Casanova

Genes Dev. 2006, 20:

Access the most recent version at doi:10.1101/gad.375706

References This article cites 39 articles, 22 of which can be accessed free at: http://genesdev.cshlp.org/content/20/13/1817.full.html\#ref-list-1

License

Email Alerting

Receive free email alerts when new articles cite this article - sign up in the box at the top Service right corner of the article or click here.

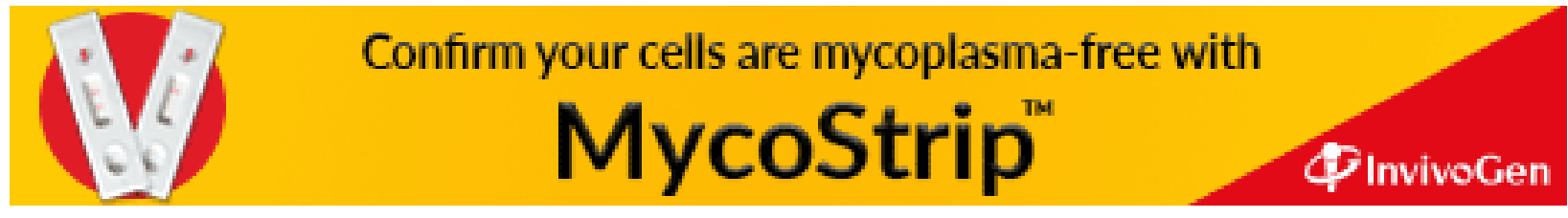

